\title{
Efeitos da Isoflavona Sobre os Sintomas Climatéricos e 0 Perfil Lipídico na Mulher em Menopausa
}

\author{
Effects of Isoflavone on Menopausal Symptoms \\ and Blood Lipids in Postmenopausal Women
}

Eliana Aguiar Petri Nahás ${ }^{1}$, Jorge Nahás Neto ${ }^{1}$, Laurival A. De Luca ${ }^{1}$, Paulo Traiman ${ }^{1}$, Anaglória Pontes ${ }^{1}$, Ivete Dalben ${ }^{2}$

\section{RESUMO}

Objetivo: avaliar os efeitos da isoflavona, do gérmen da soja, sobre os sintomas climatéricos e o perfil lipidico na mulher em menopausa.

Métodos: foi conduzido estudo prospectivo, com 50 mulheres em menopausa, divididas em: G1, usuárias de isoflavona (60 mg/dia) ( $n=25)$, e G2, placebo ( $n=25)$. Os critérios de inclusão foram FSH $>40 \mathrm{mUI} / \mathrm{mL}$ e presença de fogachos. Foram excluidas as vegetarianas, fumantes, asiáticas, portadoras de doenças gastrointestinais e usuárias de terapia de reposição hormonal. No seguimento, de seis meses, foram obtidos o indice menopausal de Kupperman (IMK), o perfil hormonal e o lipidico. Na análise estatistica, empregaram-se ANOVA, o teste $\mathrm{t}$ pareado e as provas não paramétricas de Wilcoxon e Mann-Whitney.

Resultados: os valores medianos do IMK, inicialmente iguais entre os grupos (IMK $=20$ ), reduziram-se nas usuárias de isoflavona aos 2 e 4 meses (IMK $=14$ e 9, respectivamente) e no grupo controle, apenas aos 2 meses (IMK = 15) ( $p<0,01)$. Ao final do estudo, a isoflavona foi superior ao placebo na redução dos fogachos (44\% versus $12 \%$, respectivamente). Aos seis meses, verificou-se que os valores médios de estradiol foram superiores no G1 quando comparados ao G2 (18,0 $\pm 6,7$ versus $12,3 \pm 3,8 \mathrm{ng} / \mathrm{dL})(p<0,05)$, sem alterações no FSH e $L H$. Entre as usuárias de isoflavona, houve redução de 11,8\% no LDL (de 151,5 \pm 39,2 para 133,6 $\pm 26,4 \mathrm{mg} / \mathrm{dL}$ ) e elevação de 27,3\% no $\mathrm{HDL}$ (de 44,0 $\pm 11,3$ para 56,0 $\pm 11,9 \mathrm{mg} / \mathrm{dL}$ ) ( $p<0,05$ ). Conclusões: A isoflavona, do gérmen da soja, induziu efeitos favoráveis sobre os sintomas climatéricos e o perfil lipídico, revelando-se opção interessante como terapêutica alternativa para mulheres em menopausa.

PALAVRAS-CHAVE: Menopausa. Endométrio: investigação. Perfil lipídico.

\section{Introdução}

A terapia de reposição hormonal (TRH) é recomendada para o alivio dos sintomas vasomotores, tratamento da atrofia vaginal e prevenção da osteoporose ${ }^{1}$. Apesar das conhecidas vanta-

\footnotetext{
${ }^{1}$ Disciplina de Ginecologia, Departamento de Ginecologia e Obstetricia

${ }^{2}$ Departamento de Saúde Pública, Faculdade de Medicina de Botucatu - UNESP

Correspondência:

Eliana Aguiar Petri Nahás

Departamento de Ginecologia e Obstetrícia, Faculdade de Medicina de Botucatu - UNESP

18618-000 - Rubião Júnior - Botucatu - SP

Fone: (14) 6802-6227 - Fax: (14) 6822-1933

e-mail: epetri@fmb.unesp.br
}

gens, aproximadamente $70 \%$ das mulheres cessam o tratamento após o primeiro ano ${ }^{2}$. Uma das principais causas da baixa aderência à TRH é o sangramento irregular. Outras razões incluem mastalgia, náusea, cefaléia, ganho de peso e retenção hídrica, além do medo de câncer de mama ${ }^{1}$. Estes efeitos têm desencadeado reservas em algumas mulheres, que optam por formas naturais de tratamento. Além disso, existem mulheres em que a TRH está contra-indicada, havendo pouco a se oferecer para o alivio dos sintomas climatéricos $^{3}$. Por essas razões há interesse no desenvolvimento de terapêuticas alternativas, capazes de proporcionar os beneficios da TRH sem provocar efeitos colaterais e sem contra-indicações ${ }^{1,2,4,5}$. 
Os fitoestrogênios ocorrem naturalmente em alguns vegetais, sendo estrutural e funcionalmente similares ao estradiol ${ }^{6}$. Existem quatro classes principais: as isoflavonas, encontradas na soja e seus derivados; os lignanos, nos cereais integrais e oleoginosas; os flavonóides, em algumas frutas e legumes, e os cumestranos, nos brotos de feijão e de alfafa ${ }^{7}$. Os fitoestrogênios com maior ação estrogênica são as isoflavonas, destacandose a genisteína, a daidzeína e a gliciteína, presentes no gérmen de soja. São compostos nãoesteróides, que se ligam fracamente aos receptores estrogênicos (menos que $1 \%$ da afinidade de ligação do estradiol). Apresentam ação seletiva, isto é, exibem atividade estrogênica em alguns tecidos e antiestrogênica em outros ${ }^{4,8}$.

Há evidências de que a isoflavona diminui a intensidade e a freqüência dos sintomas vasomotores em mulheres na menopausa ${ }^{9-11}$. A maioria dessas observações sobre o uso dos fitoestrogênios são epidemiológicas, muitas delas baseadas em estudos realizados em regiões de alto consumo da soja ${ }^{8}$. Menos de $20 \%$ das mulheres japonesas apresentam ondas de calor, comparado com $80 \%$ das européias, atribuindo-se, em parte, estas diferenças à dieta ${ }^{7}$. Albertazzi et al. ${ }^{3}$, em estudo duplo-cego, placebo-controlado, constataram que $60 \mathrm{~g} /$ dia de proteína isolada da soja (suplemento alimentar) foi superior ao placebo, reduzindo em $50 \%$ os sintomas vasomotores.

A dieta rica em soja parece também ser benéfica para o sistema cardiovascular, pelo efeito favorável sobre o perfil lipídico ${ }^{12-14}$. Alguns estudos demonstram elevação do HDL e redução do LDL em cerca de $10 \%^{15-18}$. Observa-se, in vitro, vasodilatação coronária e redução na agregação plaquetária e na tromboxana, resultando em menor tendência pró-trombótica ${ }^{8,19}$. Os cânceres de mama, cólon, endométrio e ovário têm menor incidência nos países asiáticos quando comparados aos ocidentais ${ }^{7}$. Em culturas de células de câncer de mama, a isoflavona demonstra efeito antiproliferativo, dose-dependente ${ }^{12}$. Outras propostas dos mecanismos relacionados com a dieta rica em soja e a possivel prevenção dos tumores malignos incluem a inibição do sistema tirosinoquinase, a supressão da angiogênese e os efeitos antioxidantes ${ }^{5}$.

Pelos numerosos beneficios relatados, a recomendação de adicionar produtos da soja à dieta seria racional ${ }^{5,20}$. A maioria dos estudos com suplementos dietéticos derivados da soja demonstraram moderado efeito estrogênico ${ }^{7}$. Entretanto, há grande dificuldade em modificar o hábito alimentar da mulher ocidental. As isoflavonas do gérmen da soja existem em forma de cápsulas, havendo poucas investigações sobre esta forma de admi- nistração. São necessários estudos adicionais para confirmar o valor desta terapêutica alternativa como eficaz e segura na menopausa ${ }^{3,20}$.

Baseado nestas considerações, o objetivo desta investigação foi avaliar os efeitos da isoflavona, do gérmen da soja, em cápsulas, sobre os sintomas climatéricos e o perfil lipídico na mulher em menopausa.

\section{Pacientes e Métodos}

Foi conduzido estudo clínico, prospectivo, randomizado, duplo-cego e placebo-controlado, constituído de 50 mulheres, em menopausa. Os critérios de inclusão foram: data da última menstruação há pelo menos 12 meses, valores de FSH superior a $40 \mathrm{mUI} / \mathrm{mL}$ e presença de sintomas de privação estrogênica, principalmente fogachos. Os critérios de exclusão: pacientes vegetarianas ou macrobióticas, asiáticas, fumantes, etilistas, história de doenças crônicas do trato gastrointestinal, uso de terapia de reposição hormonal, tamoxifeno ou antibióticos até seis meses antes do estudo. Foram efetuadas dosagens do hormônio estimulador da tiróide (TSH), tiroxina livre $\left(\mathrm{T}_{4}\right)$ e anticorpo anti-tiroperoxidase (anti-TPO) para excluir disfunções tiroideanas, que pudessem interferir na sintomatologia. Todas as pacientes assinaram o termo de consentimento livre e esclarecido. O projeto de pesquisa recebeu parecer favorável do Comitê de Ética em Pesquisa da Faculdade de Medicina de Botucatu - UNESP.

A avaliação inicial constou de anamnese, exame físico geral e ginecológico e colpocitologia oncótica. Foram obtidos os seguintes dados: idade, menarca, tempo de menopausa, paridade, hábito intestinal, peso, estatura, relação cinturaquadril e pressão arterial. A seguir, as pacientes foram distribuídas, em seqüência de numeração pré-estabelecida pelo farmacêutico, em dois grupos: $\mathrm{G} 1$, isoflavona $(n=25)$ ou $G 2$, placebo $(n=25)$. O médico examinador e as pacientes não tiveram conhecimento prévio dos referidos grupos e das diferentes numerações, apenas o farmacêutico responsável pela manipulação das cápsulas. As$\mathrm{sim}, 25$ pacientes receberam $60 \mathrm{mg}$ de isoflavona, referentes a 4 cápsulas/dia de 500 mg de gérmen de soja (Isosoy, Herborisa ${ }^{\circledR}$ ), via oral, divididas em duas tomadas, durante seis meses. Cada cápsula contém 56 mg de lipídios, 202 mg de proteína, 141 mg de carboidratos e $19 \mathrm{mg}$ de fibra, com valor energético de $2 \mathrm{kcal}$. O gérmen de soja foi extraído da mesma safra de grãos. As outras 25 pacientes receberam 4 cápsulas/dia de lactose. As cápsulas foram embaladas em frascos brancos, opa- 
cos, numerados em código pelo farmacêutico para impedir a identificação do grupo em teste pelos participantes do estudo. O tempo de seguimento foi de 6 meses, com avaliação aos 2,4 e 6 meses.

Na entrevista inicial e a cada retorno obteve-se o índice menopausal de Kupperman (IMK). Este representa a soma da conversão numérica de 11 sinais e sintomas de privação estrogênica (fogachos, parestesia, insônia, nervosismo, melancolia, vertigem, fraqueza, artralgia, cefaléia, palpitação e formigamento), graduados de 0 a 3 , a depender da intensidade leve, moderada ou intensa. Multiplicam-se por 2 a parestesia, a insônia e o nervosismo e por 4 os fogachos. Observou-se a variação ponderal pelo índice de massa corpórea $\left(\mathrm{IMC}=\right.$ peso $/$ altura $\left.^{2}\right)$. Empregaram-se os critérios da Organização Mundial da Saúde (1997), considerando-se IMC $\geq 30 \mathrm{~kg} / \mathrm{m}^{2}$ para diagnóstico de obesidade. $\mathrm{Na}$ avaliação indireta da distribuição de gordura corporal utilizou-se a relação cintura/ quadril (RCQ). Considerou-se como distribuição ginecóide quando $\mathrm{RCQ}<0,8$ e androgênica quando $\mathrm{RCQ} \geq 0,8$.

A colpocitologia hormonal foi realizada na primeira consulta e depois de seis meses. Os esfregaços foram obtidos da parede lateral da vagina, em seu terço médio. Em total de 100 células de descamação, realizou-se a contagem diferencial de parabasais, intermediárias e superficiais. Os resultados foram expressos pelo valor de maturação de Meisels ${ }^{21}$. Os exames foram interpretados pelo mesmo citopatologista, sem conhecimento dos dados das pacientes.

Foram avaliados os valores de $\mathrm{FSH}, \mathrm{LH}$ e estradiol, nos momentos basal e 6 meses. As dosagens foram realizadas pelo Sistema Elecsys ${ }^{\circledR}$ 2010 (Roche Diagnostics ${ }^{\circledR}$, Mannheim, Alemanha), que utiliza imunoensaio por eletroquimioluminescência. A sensibilidade analítica para FSH e LH foi de 0,10 mUI/mL e para estradiol, $10 \mathrm{pg} /$ $\mathrm{mL}$. Os coeficientes intra-ensaio e interensaio para FSH foram, respectivamente, de 4,2 e 5,3\%, para o $\mathrm{LH}$, de 2,6 e $5,2 \%$, e para o estradiol, de 5,6 e $6,5 \%$. Segundo critérios dos métodos, considerase menopausa valores de FSH entre 25,0 e 134,8 $\mathrm{mUI} / \mathrm{mL}, \mathrm{LH}$ entre 7,7 e $58,5 \mathrm{mUI} / \mathrm{mL}$ e estradiol entre < 10, 0 e $39,5 \mathrm{pg} / \mathrm{mL}$.

Foram avaliados os niveis séricos de triglicerídeos, colesterol total (CT), HDL e LDL, basal, aos 3 e 6 meses. As dosagens foram processadas pelo analisador bioquímico automático RAXT (Technicon ${ }^{\circledR}$, EUA). O CT, o HDL e os triglicerídeos foram quantificados pelo método colorimétrico e o LDL pela fórmula de Friedewald, subtraindo-se o valor do CT, da soma do HDL e do triglicerídeo dividido por cinco. As taxas de normalidade referidas são: CT inferior a $200 \mathrm{mg} / \mathrm{dL}$, HDL superior a 35 $\mathrm{mg} / \mathrm{dL}, \mathrm{LDL}$ inferior a $130 \mathrm{mg} / \mathrm{dL}$ e triglicerídeos inferior a $170 \mathrm{mg} / \mathrm{dL}$.

A ultra-sonografia transvaginal foi realizada para avaliação da espessura endometrial, basal e aos seis meses. Todos os exames foram obtidos pelo mesmo examinador. Utilizou-se o aparelho Sonochrome ${ }^{\circledR}\left(\mathrm{GE}^{\circledR}\right.$, EUA) com transdutor endovaginal de 7,5 MHz, para imagem morfológica de útero e ovários. A medida do endométrio, na incidência sagital, de uma camada basal a outra, foi considerada normal na menopausa quando os valores eram inferiores a $5 \mathrm{~mm}$.

Para o cálculo do tamanho amostral considerou-se intervalo de confiança de $95 \%$, a razão de um caso para um controle e a obtenção de melhora em $40 \%$ dos casos tratados. Esse cálculo foi processado pelo programa Epi-Info versão 6.04. As médias foram comparadas utilizando-se o teste $t$ pareado e a análise de variância (ANOVA), e as medianas, as provas não paramétricas de KruskalWallis e de Wilcoxon. As diferenças entre as freqüências dos casos e controles foram analisadas pelo teste do $\chi^{2}$ ou teste exato de Fisher, sempre que necessário. Os testes estatísticos foram bicaudais e o nivel de significância escolhido foi de $5 \%$ e os dados calculados com o auxílio do software SPSS versão 8.5.

\section{Resultados}

As características clínicas, colpocitológicas, ultra-sonográficas e laboratoriais iniciais, dos grupos de pacientes usuárias de isoflavona (G1) e de placebo (G2), foram submetidas à comparação estatística e estão representadas nas Tabelas 1 e 2 . Verificou-se que os grupos foram homogêneos paras as seguintes variáveis: idade, menarca, paridade, idade da menopausa, IMC, RCQ, IMK, valor de maturação e espessura endometrial (Tabela 1). Confrontando os valores séricos hormonais iniciais entre os grupos, não foram observadas diferenças estatisticamente significativas. Por outro lado, na análise do perfil lipídico, verificou-se que as pacientes do grupo isoflavona apresentaram inicialmente valores elevados de CT e LDL, quando comparadas ao grupo placebo $(\mathrm{p}<0,05)$ (Tabela 2$)$.

$\mathrm{Na}$ análise do IMK, levando-se em consideração os valores medianos basais $(\mathrm{IMK}=20, \mathrm{G} 1=$ G2), constatou-se redução significativa dos sintomas, no grupo de usuárias de isoflavona, aos dois $(\mathrm{IMK}=14)$ e aos quatro meses $(\mathrm{IMK}=9,0)$ de seguimento $(p<0,01)$, ao passo que no grupo controle, apenas aos dois meses $(\mathrm{IMK}=15)(\mathrm{p}<0,01) . \mathrm{Na}$ comparação dos grupos, houve diferença nos valo- 
res do IMK aos seis meses $(\mathrm{G} 1=8,5<\mathrm{G} 2=13,5)$ $(\mathrm{p}<0,05)$ (Tabela 3). Ao final do estudo, no interrogatório das pacientes, encontrou-se que, entre as usuárias de isoflavona, em 44\% (11/25) ocorreu desaparecimento completo dos fogachos, em 36\% (9/25) melhora parcial e em 20\% (5/25) não houve alterações. Todavia, no grupo placebo $12 \%(3 / 25)$ relatavam melhora completa das ondas de calor, $28 \%(7 / 25)$ melhora parcial e em $60 \%(15 / 25)$ não houve alterações.

Tabela 1 - Comparação das características clínicas, colpocitológicas e ultrasonográficas iniciais entre as pacientes usuárias de isoflavona (G1) $(n=25)$ e de placebo (G2) ( $n=25)$ (valores médios \pm desvios-padrão).

\begin{tabular}{lccc}
\hline Variáveis & $\begin{array}{c}\text { G1 } \\
\text { (Isoflavona) }\end{array}$ & $\begin{array}{c}\text { G2 } \\
\text { (Placebo) }\end{array}$ & Valor de $\mathbf{p}$ \\
\hline Idade (anos) & $53,7 \pm 5,4$ & $52,9 \pm 5,1$ & 0,63 \\
Menarca (anos) & $13,0 \pm 1,5$ & $13,5 \pm 1,8$ & 0,39 \\
Paridade (n ${ }^{\circ}$ de filhos) & $3,3 \pm 2,5$ & $3,0 \pm 1,3$ & 0,75 \\
Menopausa (anos) & $48,4 \pm 3,4$ & $47,9 \pm 3,5$ & 0,68 \\
IMC (kg/m²) & $28,0 \pm 5,0$ & $30,0 \pm 5,6$ & 0,23 \\
RCQ & $0,8 \pm 0,1$ & $0,8 \pm 0,1$ & 0,40 \\
IMK & $21,1 \pm 6,1$ & $20,1 \pm 6,2$ & 0,50 \\
VM (\%) & $38,8 \pm 21,7$ & $45,5 \pm 20,5$ & 0,40 \\
Endométrio (mm) & $0,4 \pm 0,1$ & $0,37 \pm 0,1$ & 0,30 \\
\hline
\end{tabular}

Dados estatisticamente não significantes (ANOVA)

IMC = İ́nice de massa corpórea

$\mathrm{RCQ}=$ Relação cintura/quadril

IMK = Índice menopausal de Kupperman

$\mathrm{VM}=$ Valor de maturação vaginal

Tabela 2 - Comparação das características laboratoriais iniciais entre as pacientes usuárias da isoflavona (G1) $(n=25)$ e o placebo (G2) $(n=25)$ (valores médios \pm desviospadrão).

\begin{tabular}{lccc}
\hline Variáveis & $\begin{array}{c}\text { G1 } \\
\text { (Isoflavona) }\end{array}$ & $\begin{array}{c}\text { G2 } \\
\text { (Placebo) }\end{array}$ & Valor de p \\
\hline FSH (mUl/ml) & $76,8 \pm 19,4$ & $75,2 \pm 28,7$ & 0,80 \\
LH (mUl/ml) & $30,7 \pm 18,5$ & $33,6 \pm 21,8$ & 0,50 \\
Estradiol (pg/ml) & $15,8 \pm 7,3$ & $14,6 \pm 6,0$ & 0,30 \\
Colesterol total (mg/dl) & $229,6 \pm 31,6$ & $203,7 \pm 25,1$ & $<0,05^{*}$ \\
HDL (mg/dl) & $44,0 \pm 11,3$ & $48,1 \pm 12,9$ & 0,28 \\
LDL (mg/dl) & $151,5 \pm 39,2$ & $130,5 \pm 23,6$ & $<0,05^{*}$ \\
Triglicerídeos (mg/dl) & $150,3 \pm 94,4$ & $139,0 \pm 68,9$ & 0,20 \\
Glicemia de jejum (mg/dl) & $102,2 \pm 28,2$ & $95,0 \pm 12,6$ & 0,30 \\
\hline
\end{tabular}

*Diferença significativa entre os grupos $(p<0,05)$ (ANOVA).

Na Tabela 4, estão apresentados os valores médios de $\mathrm{FSH}$, LH e estradiol nas pacientes usuárias de isoflavona (G1) e placebo (G2), nos momentos basal e aos 6 meses de seguimento. Verificou-se que, ao final do estudo, os valores médios de estradiol foram significantemente superiores no $\mathrm{G} 1(18,0 \pm 6,7 \mathrm{ng} / \mathrm{dl})$ quando comparados ao G2 $(12,3 \pm 3,8 \mathrm{ng} / \mathrm{dl})(\mathrm{p}<0,05)$, sem alterações no FSH e LH. Na análise do perfil lipídico, o valor médio de LDL, que inicialmente encontrava-se elevado no grupo de pacientes sob isoflavona, reduziu-se aos seis meses (de 151,5 $\pm 39,2$ para 133,6 $\pm 29,4 \mathrm{mg} / \mathrm{dl})(\mathrm{p}<0,05)$, ao passo que o HDL elevouse significativamente (de 44,0 $\pm 11,3$ para $56,0 \pm 11,9$ $\mathrm{mg} / \mathrm{dl})(\mathrm{p}<0,05)$, sem alterar os triglicerídeos. Não ocorreram mudanças desses parâmetros no grupo sob placebo (Figura 1).

Tabela 3 - Comparação dos valores do índice menopausal de Kupperman (IMK) entre as pacientes usuárias de isoflavona (G1) (n=25) e o placebo (G2) (n=25).

\begin{tabular}{lccc} 
IMK/Tempo & $\begin{array}{c}\text { G1 } \\
\text { (Isoflavona) }\end{array}$ & $\begin{array}{c}\text { G2 } \\
\text { (Placebo) }\end{array}$ & Significância * \\
\hline Basal (M1) & 20,0 & 20,0 & NS \\
& $(8-30)$ & $(10-34)$ & $\mathrm{G} 1=\mathrm{G} 2$ \\
2 meses (M2) & 14,0 & 15,0 & $\mathrm{NS}$ \\
& $(4-28)$ & $(8-22)$ & $\mathrm{G} 1=\mathrm{G} 2$ \\
4 meses (M3) & 9,0 & 13,0 & $\mathrm{NS}$ \\
& $(6-27)$ & $(5-25)$ & $\mathrm{G} 1=\mathrm{G} 2$ \\
6 meses (M4) & 8,5 & 13,5 & $\mathrm{p}<0,05$ \\
& $(5-27)$ & $(5-25)$ & $\mathrm{G} 1<\mathrm{G} 2$ \\
Significância ** & $\mathrm{p}<0,01$ & $\mathrm{p}<0,01$ & \\
\multicolumn{2}{c}{ M1>M2>(M3 = M4) } & $\mathrm{M} 1>(\mathrm{M} 2=\mathrm{M} 3=\mathrm{M} 4)$
\end{tabular}

Os resultados são expressos em mediana, com os valores mínimo e máximo entre parênteses. * Diferença significativa entre os grupos (teste de Mann-Whitney)

** Diferença significativa em relação ao valor basal dentro do grupo (teste de Wilcoxon) $M=$ Momentos

NS = Não significativo

Tabela 4 - Comparação dos valores de FSH $(\mathrm{mUl} / \mathrm{mL}), \mathrm{LH}(\mathrm{mUl} / \mathrm{mL})$ e estradiol $\left(\mathrm{E}_{2}, \mathrm{pg} / \mathrm{mL}\right)$ entre as pacientes usuárias de isoflavona ( $\mathrm{G} 1)(\mathrm{n}=25)$ e de placebo $(\mathrm{G} 2)(\mathrm{n}=25)$ (valores médios \pm desvios-padrão).

\begin{tabular}{|c|c|c|c|c|}
\hline \multirow{2}{*}{$\begin{array}{l}\text { Momentos/ } \\
\text { Variáveis }\end{array}$} & \multicolumn{2}{|c|}{ Basal } & \multicolumn{2}{|c|}{6 meses } \\
\hline & G1 & G2 & G1 & G2 \\
\hline $\mathrm{FSH}(\mathrm{mUl} / \mathrm{ml})$ & $76,8 \pm 13,4$ & $75,2 \pm 28,7$ & $77,7 \pm 19,6$ & $82,5 \pm 30,7$ \\
\hline $\mathrm{LH}(\mathrm{mUl} / \mathrm{ml})$ & $30,7 \pm 8,51$ & $33,9 \pm 21,8$ & $30,6 \pm 9,7$ & $34,4 \pm 16,7$ \\
\hline$E_{2}(n g / d l)$ & $15,8 \pm 7,3$ & $14,6 \pm 6,0$ & $18,0 \pm 6,7^{* *}$ & $12,3 \pm 3,8$ \\
\hline
\end{tabular}

Aos seis meses, a mediana do valor de maturação (\%) das células vaginais manteve-se inalterada nas pacientes com isoflavona, quando comparado ao valor basal, ao passo que, no grupo placebo, houve redução significativa (de 54 para $48 \%)(p<0,05)$. Durante o seguimento não se alteraram os valores do IMC, RCQ e a pressão arterial, em ambos os grupos. Na ultra-sonografia transvaginal, não se observou variação nos valores médios da espessura endometrial. Todavia, três pacientes, duas sob isoflavona e uma com placebo, apresentaram discreto sangramento vaginal, durante o estudo, com medidas do endométrio superiores a $5 \mathrm{~mm}$. Realizou-se histeroscopia diagnóstica, que evidenciou dois casos de endométrio atrófico e um de pólipo endometrial. 

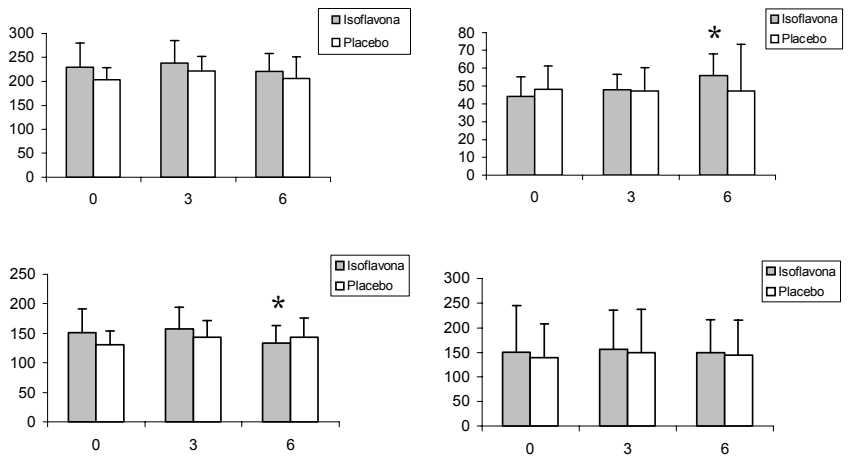

**Diferença significativa na comparação entre os grupos $(p<0,05)$ (ANOVA)

*Diferença significativa em relação ao valor basal dentro do grupo $(p<0,05)$ (teste $t$ pareado)

Figura 1 - Comparação entre os valores iniciais e finais, entre as pacientes usuárias de isoflavona(G1) ( $\mathrm{n}=25)$ ede placebo(G2)(n=25), das dosagens de colesterol total (CT, mg/dL, HDL(mg/dL), LDL(mg/dL)e triglicerídeos (TG, mg/dL) (valores médios \pm desvios-padrão).

\section{Discussão}

As isoflavonas encontram-se na forma glicosilada na natureza, biologicamente inativas. Após ingestão, ocorre complexo mecanismo enzimático de conversão no trato gastrointestinal, resultando na formação de fenóis heterocíclicos estruturalmente similares ao 17 - $\beta$-estradiol ${ }^{6,7}$. Decorrente deste fator, as concentrações dos diferentes metabólitos dos fitoestrogênios e seus efeitos clínicos têm variação individual mesmo quando controlada a quantidade de isoflavona administrada, sendo dificil estabelecer a dose ideal. Recomendam-se de 20 a $100 \mathrm{mg} / \mathrm{dia}^{2,20}$. Nesta pesquisa empregou-se dose de $60 \mathrm{mg} /$ dia de gérmen da soja, na forma glicosilada natural, de maior biodisponibilidade. Há poucas pesquisas utilizando essa forma de apresentação da isoflavona. Os estudos em sua maioria utilizam cápsulas de extrato de isoflavona da soja, de menor biodisponibilidade ou suplementação de soja na dieta. As concentrações de isoflavona na alimentação à base de soja também são variáveis, dependendo da qualidade do grão, das condições de crescimento, do solo e do processamento. Em $60 \mathrm{~g}$ de soja encontram-se, em média, $70 \mathrm{mg}$ de isoflavonas, sendo $40 \mathrm{mg}$ de genisteína e $30 \mathrm{mg}$ de daidzeína ${ }^{3}$.

Neste estudo, aos dois meses, constatou-se redução na freqüência de problemas típicos do climatério, demonstrados pela diminuição significativa do IMK em ambos os grupos, sugerindo efeito placebo inicial. Porém, aos quatro e seis meses de seguimento, a melhora dos sintomas foi observada apenas entre as usuárias de isoflavona. $\mathrm{O}$ fogacho é fenômeno comum na transição para a menopausa, relacionado ao hipoestrogenismo e contemplado pelo IMK. No grupo de isoflavona ocorreu alívio completo das ondas de calor em $44 \%$ das pacientes contra $12 \%$ sob placebo. Esses resultados estão em concordância com outros autores, que notaram melhora dos sintomas vasomotores com suplementação dietética de soja ${ }^{3,9,21}$. Scambia et al. ${ }^{10}$ utilizando cápsulas de isoflavona da soja (50 mg/dia), evidenciaram melhora significativa das ondas de calor. Em recente pesquisa, Han et al. ${ }^{11}$ demonstraram redução significante do IMK, após 4 meses, com $100 \mathrm{mg}$ de isoflavona de extrato da soja, em cápsulas.

O valor médio de maturação vaginal não se alterou em 6 meses entre as usuárias de isoflavona, sugerindo manutenção do trofismo vaginal. Por outro lado, nas pacientes que usaram placebo encontrou-se redução significante do valor médio de maturação vaginal. Há muita controvérsia entre os estudos, o que torna confusa a atribuição de efeitos benéficos dos fitoestrogênios sobre a citologia vaginal. Em estudo pioneiro, Wilcox et al. ${ }^{22}$ constataram melhora da maturação vaginal com $45 \mathrm{~g}$ de soja na dieta. Dalais et al. ${ }^{9}$ observaram aumento significativo no índice de maturação vaginal no grupo de mulheres em menopausa submetidas a suplementação alimentar de soja. Porém, outros estudos não demonstraram melhora significativa da mucosa vaginal $1^{4,10,11,23}$.

Dislipidemia, hipertensão arterial e obesidade central estão associadas com alto risco para doença cardiovascular, para mulheres na menopausa. Não se observaram o IMC e a RCQ nos seis meses de uso da isoflavona ou placebo. Também, não se observou variações na pressão arterial, apesar de $40 \%(10 / 25)$ no grupo de isoflavona e $32 \%(8 / 25)$ no grupo placebo serem hipertensas crônicas controladas, usuárias de terapêuticas anti-hipertensivas. de Kleijn et al. ${ }^{14}$ avaliaram a dieta de 939 mulheres em menopausa, participantes do Framingham Offspring Study. Demonstraram que, nas consumidoras de dieta rica em fitoestrogênios, a RCQ e os niveis de triglicerídeos foram significativamente menores, quando comparadas àquelas com dieta pobre em soja. Concluem que a elevada ingestão de fitoestrogênios na menopausa parece associar-se com favorável perfil metabólico para doença cardiovascular.

Nas pacientes do grupo de isoflavona, os valores médios iniciais de CT e LDL foram significativamente superiores aos do grupo que recebeu placebo. Após seis meses, entre as usuárias de 60 $\mathrm{mg}$ isoflavona houve redução significativa de $11,8 \%$ nos valores médios de LDL e elevação de $27,3 \%$ no HDL, com pequena diminuição no colesterol total. Anderson et al. ${ }^{15}$ realizaram meta-análise avaliando o perfil lipídico em 38 estudos com dieta rica em soja. Encontraram redução média de $12,9 \%$ de LDL, porém sem alterações de HDL. Entretanto, outras pesquisas de- 
monstraram aumentos variáveis de 3,7 a $28,6 \%$ nos valores médios de $\mathrm{HDL}^{16-18}$. Han et al. ${ }^{11}$, com $100 \mathrm{mg} /$ dia de isoflavona da soja em cápsulas, obtiveram redução do CT e LDL, quando comparado ao placebo, sem variações de HDL e triglicerídeos. Dewell et al. ${ }^{13}$, com suplementação de $150 \mathrm{mg}$ de soja, em pacientes com hipercolesterolemia, não constataram mudanças no perfil lipídico.

Ao final desta investigação, evidenciou-se elevação significativa dos valores médios de estradiol plasmático entre as pacientes que receberam isoflavona quando comparadas às do grupo placebo, mantendo-se, porém, em niveis inferiores a $20 \mathrm{pg} / \mathrm{mL}$. As isoflavonas, compostos nãoesteróides, parecem ter efeitos hormonais do tipo estrogênio quando ingeridas em doses elevadas ${ }^{24}$. Cassidy et al. ${ }^{25}$ demonstraram aumento do estradiol em mulheres na perimenopausa usando $45 \mathrm{mg} /$ dia de isoflavona. Duncan et al. ${ }^{24}$ encontraram redução nos valores de estrona, sem alterações de estradiol, em mulheres na menopausa com dieta rica em isoflavona. Em outros estudos não se observaram mudanças nas dosagens hormonais ${ }^{10,11,18}$.

No presente estudo não se constataram alterações na espessura do endométrio, pela ultrasonografia transvaginal, em ambos os grupos. Pesquisas avaliando os efeitos da isoflavona sobre o endométrio têm mostrado que o uso de suplementos alimentares ou de cápsulas, na menopausa, não altera a espessura endometrial ${ }^{10,11,24,26}$. Os fitoestrogênios parecem ter ação antiestrogênica e antiproliferativa no tecido endometrial ${ }^{24}$.

Não há ainda evidências suficientes para recomendar o uso dos fitoestrogênios como substituto da TRH convencional ${ }^{1,2,12}$. Todavia, nesta investigação a isoflavona, em cápsulas de gérmen da soja, na dose de $60 \mathrm{mg} /$ dia, induziu efeitos favoráveis sobre os sintomas climatéricos e o perfil lipídico, revelando-se opção interessante como terapêutica alternativa para mulheres em menopausa.

\section{ABSTRACT}

Purpose: to evaluate the effects of soy germ isoflavone on menopausal symptoms and blood lipids in postmenopausal women.

Methods: a prospective study was performed on 50 women, randomly divided into two groups: 25 women on soy germ isoflavones (60 mg/day, capsules) (G1) and 25 women on placebo (G2). Inclusion criteria: women with hot flushes and $F S H>40 \mathrm{mIU} / \mathrm{mL}$, non-vegetarian, non-smoker, non-Asiatic, not in use of hormone replacement therapy and without disease of the gastrointestinal tract. For six months, the menopausal Kupperman index (MKI) and hormonal and lipid profiles were assessed. For statistical analysis, ANOVA, $\mathrm{t}$ test and the non-parametric Kruskal-Wallis and MannWhitney tests were used.

Results: the median MKI values, initially similar in both groups, decreased in G1 at two and four months (MKI = 14 and 9, respectively), and in $G 2$ at two months $(M K I=15)$ $(p<0.01)$. At six months, isoflavone was significantly superior to placebo in reducing hot flushes (44 versus $12 \%$, respectively). At the end of the study, in the isoflavone group,

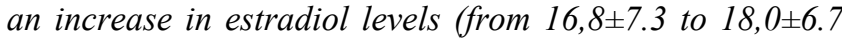
$n g / d L)(p<0.05)$ was observed, with no alteration in FSH, $L H$ and vaginal cytology; there was also a reduction of $11.8 \%$ in $L D L$ (from 151.5 39.2 for $133,6 \pm 26.4 \mathrm{mg} / \mathrm{dL}$ ) and a $H D L$ increase of $27.3 \%$ (from $44.0 \pm 11.3$ to $56.0 \pm 11.9 \mathrm{mg} / \mathrm{dL}$ ) $(p<0.05)$.

Conclusions: Soy germ isoflavone induced favorable effects on menopausal symptoms and lipid profile, showing to be an interesting option for alternative therapy in postmenopausal women.

KEYWORDS: Menopause. Hot flushes. Lipid profile.

\section{Conflitos de interesse:}

A medicação Isosoy ${ }^{\circledR}$ foi gentilmente cedida por Herborisa ${ }^{\circledR}$.

\section{Referências}

1. Glazier MG, Bowman MA. A review of the evidence for the use of phytoestrogens as a replacement for traditional estrogen replacement therapy. Arch Intern Med 2001; 161:1161-72.

2. Elkind-Hirsch K. Effect of dietary phytoestrogens on hot flushes: can soy-based proteins substitute for traditional estrogen replacement therapy? Menopause 2001; 8:154-6.

3. Albertazzi P, Pansini F, Bonaccorsi G, Zanotti L, Forini E, De Aloysio D. The effect of dietary soy supplementation on hot flushes. Obstet Gynecol 1998; 91:6-11.

4. Baker VL, Leitman D, Jaffe RB. Selective estrogen receptor modulators in reproductive medicine and biology. Obstet Gynecol Surv 2000; 55(Supp1 2):S2 1-47.

5. Kass-Annese B. Alternative therapies for menoapause. Clin Obstet Gynecol 2000; 43:162-83.

6. Mackey R, Eden J. Phytoestrogens and the menopause. Climacteric 1998; 1:302-8.

7. Murkies AL, Wilcox G, Davis SR. Phytoestrogens. J Clin Endocrinol Metab 1998; 83:297-303. 
8.Lissin LW, Cooke JP. Phytoestrogens and cardiovascular health. J Am Coll Cardiol 2000, 35:1403-10.

9. Dalais FS, Rice GE, Wahlqvist ML, et al. Effects of dietary phytoestrogens in postmenopausal women. Climacteric 1998; 1:124-9.

10.Scambia G, Mango D, Signorile PG, et al. Clinical effects of a standardized soy extract in postmenopausal women: a pilot study. Menopause 2000; 7:105-11.

11.Han KK, Soares JM Jr, Haidar MA, de Lima GR, Baracat EC. Benefits of soy isoflavone therapeutic regimen on menopausal symptoms. Obstet Gynecol 2002; 99:389-94.

12.Davis SR. Phytoestrogen therapy for menopausal symptoms? There's no good evidence that it's any better than placebo. BMJ 2001; 323:354-5.

13. Dewell A, Hollenbeck CB, Bruce B. The effects of soy-derived phytoestrogens on serum lipids and lipoproteins in moderately hypercholesterolemic postmenopausal women. J Clin Endocrinol Metab 2002; 87:118-21.

14.de Kleijn MJ, van der Schouw YT, Wilson PW, Grobbee DE, Jacques PF. Dietary intake of phytoestrogens is associated with a favorable metabolic cardiovascular risk profile in postmenopausal US women: the Framingham study. J Nutr 2002; 132:276-82.

15.Anderson JW, Johnstone BM, Cook-Newell ME. Meta-analysis of the effects of soy protein intake on serum lipids. N Engl J Med 1995; 333:276-82.

16.Potter SM, Baum JA, Teng H, Stillman RJ, Shay NF, Erdman JW Jr. Soy protein and isoflavones: their effects on blood lipids and bone density in postmenopausal women. Am J Clin Nutr 1998; 68:1375S-1379S.
17.Clifton-Bligh PB, Baber RJ, Fulcher GR, Nery ML, Moreton T. The effect of isoflavone extracted from red clover (Rimostil) on lipid and bone metabolism. Menopause 2001; 8:259-65.

18.Scheiber MD, Rebar RW. Isoflavones and postmenoausal bone health: a viable alternative to estrogen therapy? Menopause 1999; 6:233-41.

19.Nestel PJ, Pomeroy S, Kay S, et al. Isoflavones from red clover improve systemic arterial compliance but not plasma lipds in menoapusal women. J Clin Endocrinol Metab 1999; 84:895-8.

20.Brouns F. Soya isoflavones: a new and promising ingredient for the health foods sector. Food Res Int 2002; 35:187-93.

21. Meisels A. The maturation value. Acta Cytol 1967; 11:249-53.

22.Wilcox G, Wahlqvist ML, Burger HG, Medley G. Oestrogenic effects of plant foods in postmenopausal women. BMJ 1990; 301:905-6.

23.Nagata C, Shimizu H, Takami R, Hayashi M, Takeda N, Yasuda K. Hot flushes and other menopausal symptoms in relation to soy product intake in Japanese women. Climacteric 1999; 2:6-12.

24.Duncan AM, Underhill KE, Xu X, Lavalleur J, Phipps WR, Kurger MS. Modest hormonal effects of soy isoflavones in postmenopausal women. J Clin Endocrinol Metab 1999; 84:3479-84.

25.Cassidy A, Bingham S, Setchell KD. Biological effects of diet of soy protein rich in isoflavones on the menstrual cycle of premenopausal women. Am J Clin Nutr 1994; 60:333-40.

26. Hale GE, Hughes CL, Cline JM. Endometrial cancer: hormonal factors, the perimenopausal "window of risk", and isoflavones. J Clin Endocrinol Metab 2002; 87:3-15.

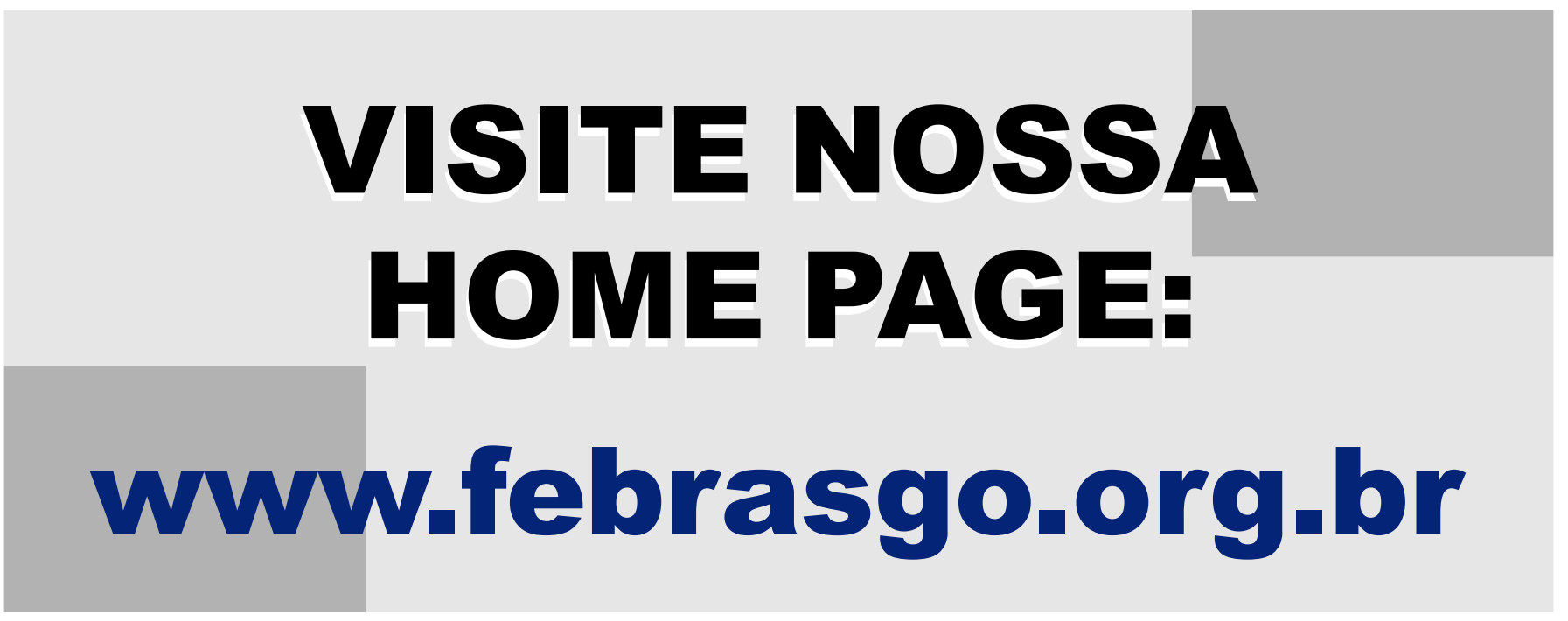

